\title{
Clinical Etiology of Hypermetabolic Pelvic Lesions in Postoperative Positron Emission Tomography/Computed Tomography for Patients With Rectal and Sigmoid Cancer
}

\author{
Yun Hee Kang ${ }^{1}$, Eunji $\mathrm{Han}^{2}$, Geon Park ${ }^{3}$ \\ ${ }^{1}$ Department of Nuclear Medicine, Eulji University Hospital, Daejeon; Departments of ${ }^{2}$ Nuclear Medicine and ${ }^{3}$ Radiology, The Catholic \\ University of Korea, Daejeon St. Mary's Hospital, Daejeon, Korea
}

Purpose: The purpose of this study was to present various clinical etiologies of hypermetabolic pelvic lesions on postoperative positron emission tomography/computed tomography (PET/CT) images for patients with rectal and sigmoid cancer. Methods: Postoperative PET/CT images for patients with rectal and sigmoid cancer were retrospectively reviewed to identify hypermetabolic pelvic lesions. Positive findings were detected in 70 PET/CT images from 45 patients; 2 patients who were lost to follow-up were excluded. All PET findings were analyzed in comparison with contrast-enhanced CT.

Results: A total of 43 patients were classified into 2 groups: patients with a malignancy including local recurrence $(n=30)$ and patients with other benign lesions $(n=13)$. Malignant lesions such as a local recurrent tumor, peritoneal carcinomatosis, and incidental uterine malignancy, as well as various benign lesions such as an anastomotic sinus, fistula, abscess, reactive lymph node, and normal ovary, were observed.

Conclusion: PET/CT performed during postoperative surveillance of rectal and sigmoid colon cancer showed increased fluorodeoxyglucose uptake not only in local recurrence, but also in benign pelvic etiologies. Therefore, physicians need to be cautious about the broad clinical spectrum of hypermetabolic pelvic lesions when interpreting images.

\section{Keywords: Colorectal neoplasms; Fluorodeoxyglucose F18; Local neoplasm recurrence; Positron-emission tomography}

\section{INTRODUCTION}

Despite the progress in surgical and adjuvant therapeutic modalities in the treatment of patients with colorectal cancer, metastasis or local recurrence occurs in $30 \%-50 \%$ of all patients within 2 years of surgery. Pelvic recurrence occurs in as many as $30 \%$ of all cases [1]. Radiologic tools, such as computed tomography (CT) and magnetic resonance imaging (MRI), are used to detect recurrence, and more recently, $18 \mathrm{~F}$-fluorodeoxyglucose $\left({ }^{18} \mathrm{~F}-\mathrm{FDG}\right)$ positron emission tomography/CT (PET/CT) has shown excel-

Received: May 25, 2016 • Accepted: September 21, 2017

Correspondence to: Geon Park, M.D.

Department of Radiology, The Catholic University of Korea, Daejeon Saint Mary's Hospital, 64 Daeheung-ro, Jung-gu, Daejeon 34943, Korea

Tel: +82-42-220-9639, Fax: +82-42-220-9087

E-mail: greempark@gmail.com

ORCID code: https://orcid.org/0000-0002-8486-3924

(C) 2018 The Korean Society of Coloproctology

This is an open-access article distributed under the terms of the Creative Commons Attribution NonCommercial License (http://creativecommons.org/licenses/by-nc/4.0) which permits unrestricted noncommercial use, distribution, and reproduction in any medium, provided the original work is properly cited. lent diagnostic performance in the detection of local recurrence and metastasis in patients with colorectal cancer. However, PET images show increased FDG uptake not only in locally recurrent malignancies and metastases but also for other conditions, such as inflammation $[2,3]$. In this study, we present the clinical etiology of hypermetabolic pelvic lesions in postoperative PET/CT images of patients with rectal and sigmoid colon cancer.

\section{METHODS}

Postoperative PET/CT and contrast-enhanced CT (CECT) images for patients with rectal and sigmoid cancer who had undergone an anterior resection or a lower anterior resection between January 2011 and April 2015 were reviewed retrospectively to identify hypermetabolic pelvic lesions. A total of 894 PET/CT procedures were performed for restaging, therapeutic response monitoring, or postoperative surveillance in 410 patients. The median time interval between PET/CT and surgery was 36 months (range, 1.5-184 months). Positive findings were detected in 70 PET/CT images from 45 patients, and all PET findings were 
Volume 34, Number 2, 2018

analyzed in comparison with CECT findings.

All ${ }^{18} \mathrm{~F}$-FDG PET/CT imaging was performed on a Discovery 690 PET/CT scanner (GE Medical System, Waukesha, WI, USA). Blood glucose levels before scanning were checked and were $<180$ $\mathrm{mg} / \mathrm{dL}$. PET/CT images were acquired 1 hour after injection of $5.55 \mathrm{MBq}$ of FDG per kg body weight. A helical nonenhanced CT scan was performed from the top of the skull base to the midthigh with normal breathing. Immediately after CT, PET was performed covering the same axial-field views of the body. PET emission data were acquired at 2 minutes per bed position. PET images were generated using standard 3-dimensional VUE point FX (VPFX, GE Healthcare, Chicago, IL, USA) time-of-flight reconstruction algorithms with CT-based attenuation correction. CECT scans were performed using two multidetector CT scanners (SOMATOM Definition and SOMATOM Definition AS+,
Siemens Medical Solution, Forchheim, Germany) with contrast enhancement (Optiray, Guerbet).

Two nuclear medicine physicians and a radiologist independently reviewed the PET/CT and CECT images, respectively. We opted to use visual inspection rather than an absolute standardized uptake value to define a hypermetabolic focus. Pelvic recurrence was classified according to the location of the lesion [4].

As it is a retrospective study that does not collect personally identifiable information, it is subject to an exemption from IRB review.

\section{RESULTS}

Forty-five of the 410 patients (11\%) had hypermetabolic pelvic lesions on PET/CT imaging. Four recurrent lesions and 6 benign
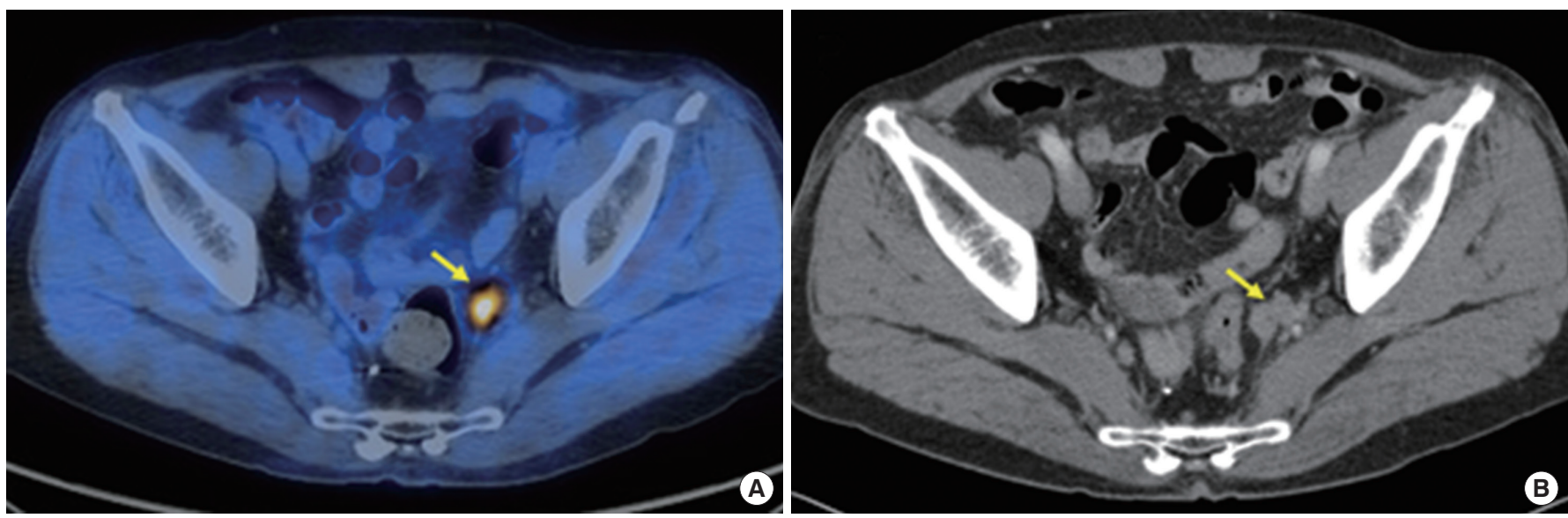

Fig. 1. A 63-year-old male had undergone a laparoscopic low anterior resection due to rectal cancer 1 year earlier. (A) A recurrent tumor in the left pelvic side wall was detected in the positron emission tomography/computed tomography (CT) fusion image as increased fluorodeoxyglucose uptake in the tumor (arrow) and (B) presented as an enhancing tumor (arrow) in the contrast-enhanced CT image.
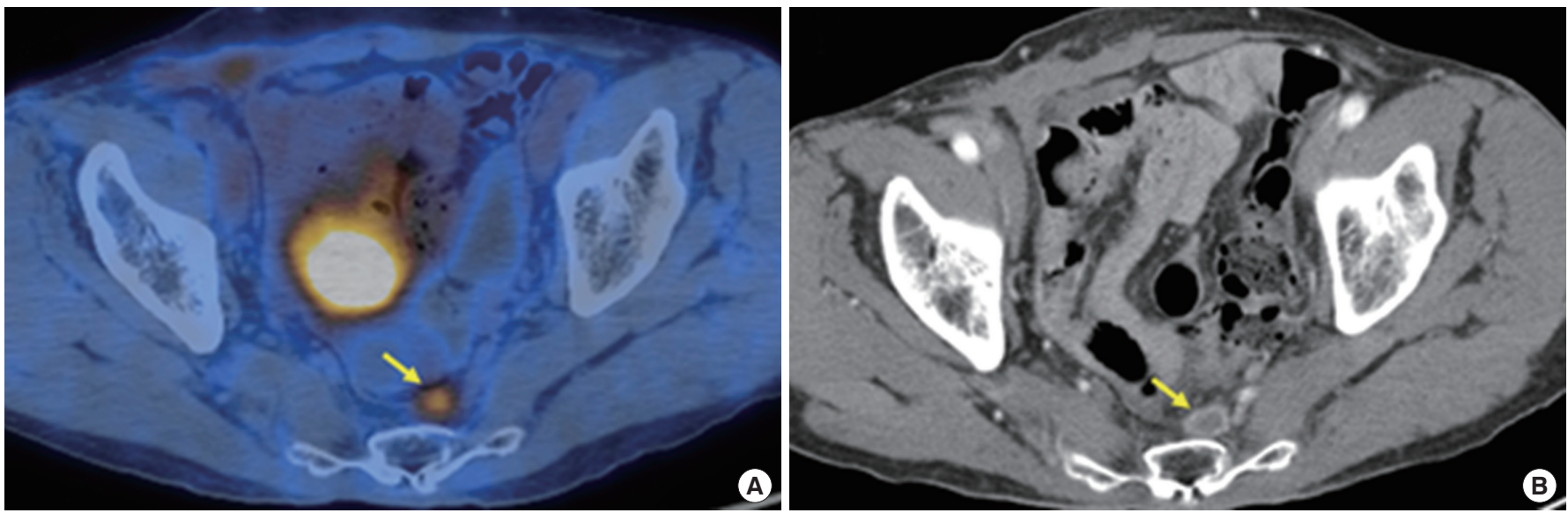

Fig. 2. A 71-year-old male had undergone a laparoscopic low anterior resection due to rectal cancer 3 years earlier. (A) A recurrent tumor (arrow) with increased fluorodeoxyglucose uptake in the presacral fascia was detected in the positron emission tomography/computed tomography (CT) fusion image. (B) The lesion was seen as a peripheral enhancing tumor (arrow) in the contrast-enhanced CT image. 

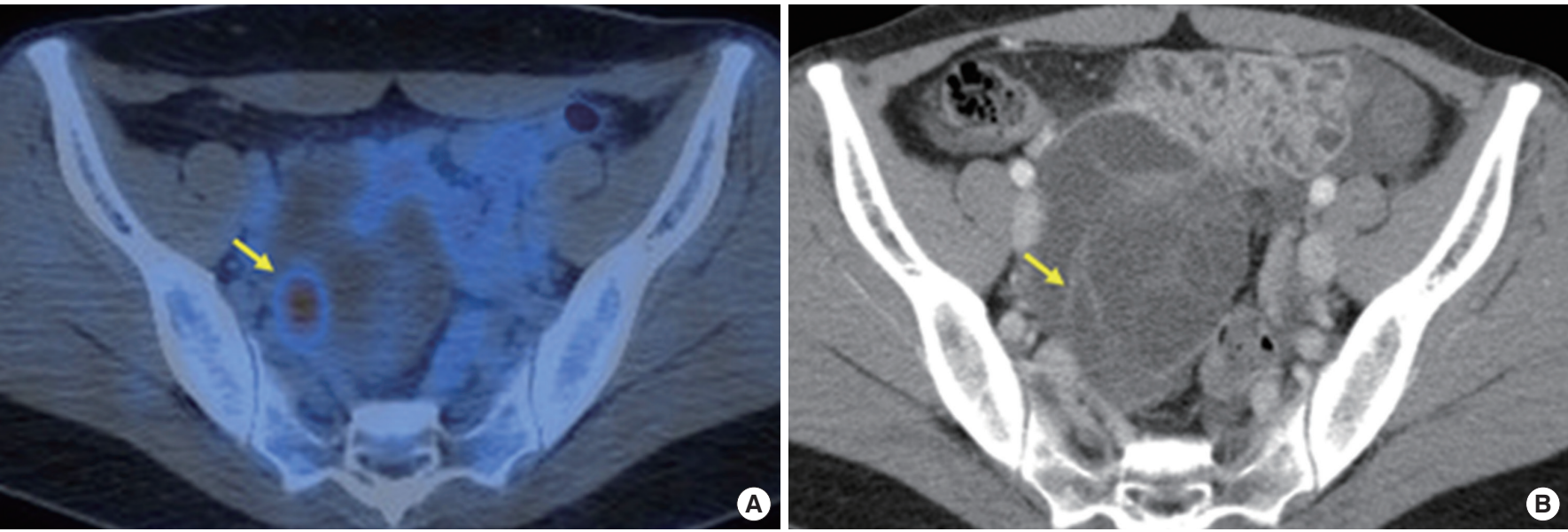

Fig. 3. A 41-year-old female had undergone a laparoscopic anterior resection due to sigmoid colon cancer 1 year earlier. (A) A multilocular cystic mass in the right ovary with increased fluorodeoxyglucose uptake at the solid portion of the tumor (arrow) was identified in the positron emission tomography/computed tomography (CT) fusion image and (B) presented as a solid, cystic tumor with multiple enhancing septa (arrow) in the contrast-enhanced CT image. Following surgical resection, pathology confirmed ovarian metastasis.

Table 1. Malignant and benign etiologies of pelvic ${ }^{18} \mathrm{~F}-\mathrm{FDG}$ uptake on $\mathrm{PET} / \mathrm{CT}$

\begin{tabular}{lcc}
\hline Lesion & No. of patients & SUVmax, mean (range) \\
\hline Malignant lesions $(\mathrm{n}=30)$ & & \\
\hline Local recurrence & & \\
$\quad$ Presacral fascia & 7 & $7.6(4-12.9)$ \\
$\quad$ Side wall & 7 & $10.5(3.5-18.6)$ \\
Lymph nodes & 3 & $7(4.4-11)$ \\
Pelvic floor & 2 & $7.7(4.9-11.2)$ \\
Ovary & 1 & $4.1(3.9-4.2)$ \\
Bladder & 7 & $8.42^{\mathrm{a}}$ \\
Peritoneal carcinomatosis & 1 & $7.9(5.5-12)$ \\
Another malignancy & & $11.5(8-13)$ \\
Benign lesions (n=13) & 8 & $7.5(4.5-12.5)$ \\
Anastomotic sinus & 3 & $6.5(4-8.5)$ \\
Lymph nodes & 2 & $7.5(6.3-9.8)$ \\
Ovary & 1 & $5.7(4-7.3)$ \\
Perirectal inflammation & 1 & $8.1(8.0-8.1)$ \\
Fistula & 1 & $11.2^{\mathrm{a}}$ \\
\hline Abscess & & \\
\hline
\end{tabular}

Forty-three patients had hypermetabolic pelvic lesions. The number of pelvic lesions does not total 43 because some patients had more than one etiology.

${ }^{18} \mathrm{~F}-\mathrm{FDG}, 18 \mathrm{~F}$-fluorodeoxyglucose; PET/CT, positron emission tomography/computed tomography; SUVmax, maximum standardized uptake value. aMean.

lesions were confirmed by surgery or biopsy. Other lesions were confirmed by further imaging studies (CECT, MRI) and at least 12 months of clinical follow-up. Two patients who were lost to follow-up were excluded. Thus, data from 43 patients were in- cluded in this study. The histologic subtype was an adenocarcinoma in all 43 patients: rectal cancer $(\mathrm{n}=34)$, rectosigmoid colon cancer $(n=5)$, and sigmoid colon cancer $(n=4)$. These patients (27 male, 16 female patients; age range, 35-95 years; mean age, $63.1 \pm 12.9$ years) were classified into 2 groups: patients with malignant lesions $(\mathrm{n}=30)$ including local recurrence (Figs. 1-3), and patients with other benign lesions $(n=13)$. Recurrence $(n=23$, $5.6 \%)$ was classified according to Sinaei et al. [4] by using lesion location. These results are detailed in Table 1. Demographic and clinicopathologic features are described in Table 2.

\section{DISCUSSION}

Local recurrence of colorectal cancer after resection is associated with serious morbidity and mortality, and early detection at an operable stage is crucial [1]. According to a study by Tagliacozzo and Accordino [5], pelvic recurrence after surgical treatment of patients with rectal and sigmoid cancer occurred in $16 \%$ of those patients, and $80 \%$ of those recurrences occurred within 2 years of the surgery. For the detection of such recurrences, several diagnostic tools, such as hemoglobin and carcinoembryonic antigen (CEA) testing, CT, MRI, endoscopic ultrasound, endoscopy, PET, etc., are used $[2,6]$.

The reported accuracy of FDG PET for detecting pelvic recurrences of colorectal cancer ranges from $74 \%$ to $96 \%$ [7]. Makis et al. [8] reported that PET/CT was superior to serum CEA for the detection of recurrences. Fiocchi et al. [2] reported that PET/CT showed a high sensitivity of $94.4 \%$ for the evaluation of patients with suspected local recurrence of colorectal cancer. In our study, in addition to local recurrences, other malignant lesions, such as peritoneal carcinomatosis $(\mathrm{n}=7)$ and an incidental uterine carcinoma $(n=1)$, showed increased FDG uptake. Although PET is 
Volume 34, Number 2, 2018

Table 2. Demographic and clinicopathologic information of the patients

\begin{tabular}{|c|c|c|}
\hline Characteristic & Malignant lesion & Benign lesion \\
\hline Age (yr), mean (range) & $64.4(41-95)$ & $60.1(35-76)$ \\
\hline \multicolumn{3}{|l|}{ Sex } \\
\hline Male & 19 & 8 \\
\hline Female & 11 & 5 \\
\hline \multicolumn{3}{|l|}{ Primary tumor location } \\
\hline Rectum & 22 & 12 \\
\hline Rectosigmoid & 4 & 1 \\
\hline Sigmoid colon & 4 & 0 \\
\hline \multicolumn{3}{|l|}{ Histological type } \\
\hline Adenocarcinoma & 30 & 13 \\
\hline Nonadenocarcinoma & 0 & 0 \\
\hline \multicolumn{3}{|c|}{ Primary tumor, pathological stage } \\
\hline $\mathrm{T} 1$ & 2 & 0 \\
\hline $\mathrm{T} 2$ & 3 & 5 \\
\hline T3 & 20 & 5 \\
\hline T4 & 5 & 3 \\
\hline \multicolumn{3}{|c|}{ Lymph node involvement, pathological stage } \\
\hline NO & 12 & 7 \\
\hline N1 & 7 & 6 \\
\hline N2 & 11 & 0 \\
\hline \multicolumn{3}{|l|}{ Distant metastasis } \\
\hline M0 & 21 & 13 \\
\hline M1 & 9 & 0 \\
\hline \multicolumn{3}{|l|}{ CEA (ng/mL) } \\
\hline Within the normal range & 13 & 12 \\
\hline Elevated & 17 & 1 \\
\hline
\end{tabular}

Values are presented as number of patients unless otherwise indicated. CEA, carcinoembryonic antigen. regarded as being highly sensitive, it shows increased FDG uptake in other benign conditions, such as inflammation; it can also show physiologic uptake in muscle, fat, and bowel tissue [2, 9]. In our study, 13 patients showed a variety of hypermetabolic, benign etiologies. An anastomotic sinus was detected in 8 patients, and benign reactive lymph nodes, normal ovaries, perirectal inflammations, perianal fistulae, and abscesses were detected in others (Figs. 4, 5).

Anastomotic leakage as a postsurgical complication after bowel resection surgery can eventually evolve into a presacral sinus, fistula, and/or abscess [10]. To our knowledge, no study has addressed the PET/CT findings for an anastomotic sinus or fistula. The mechanism of FDG uptake is uncertain in patients with an anastomotic leakage, but it may be due to the inflammatory processes associated with this complication [11]. In prospective studies, Lerman et al. [12] and Nishizawa et al. [13] reported physiological ovarian FDG uptake in premenopausal women during the ovulation phase of the menstrual cycle. Thus, a differential diagnosis of normal ovaries and malignant ovarian lesions based only on hypermetabolism is problematic $[12,13]$. Therefore, other imaging studies or a clinical interview about the menstrual cycle will be needed when diagnosing hypermetabolic ovarian lesions [13].

In our study, we obtained CECT images as a way to obtain further anatomical and morphological information, which provided the pattern of contrast enhancement and lesion characterization and allowed differentiation of benign hypermetabolic lesions from malignancy and accurate diagnosis of pathology. Detailed comparisons to the anatomical and the morphological structures on the CECT images, in addition to those on the nonenhanced PET/CT images, should assist in the accurate identification of hypermetabolic pelvic lesions on PET images.

Our study has several limitations. First, the selected population included only patients with rectal and sigmoid cancer who underwent PET/CT for postoperative surveillance imaging. Second,
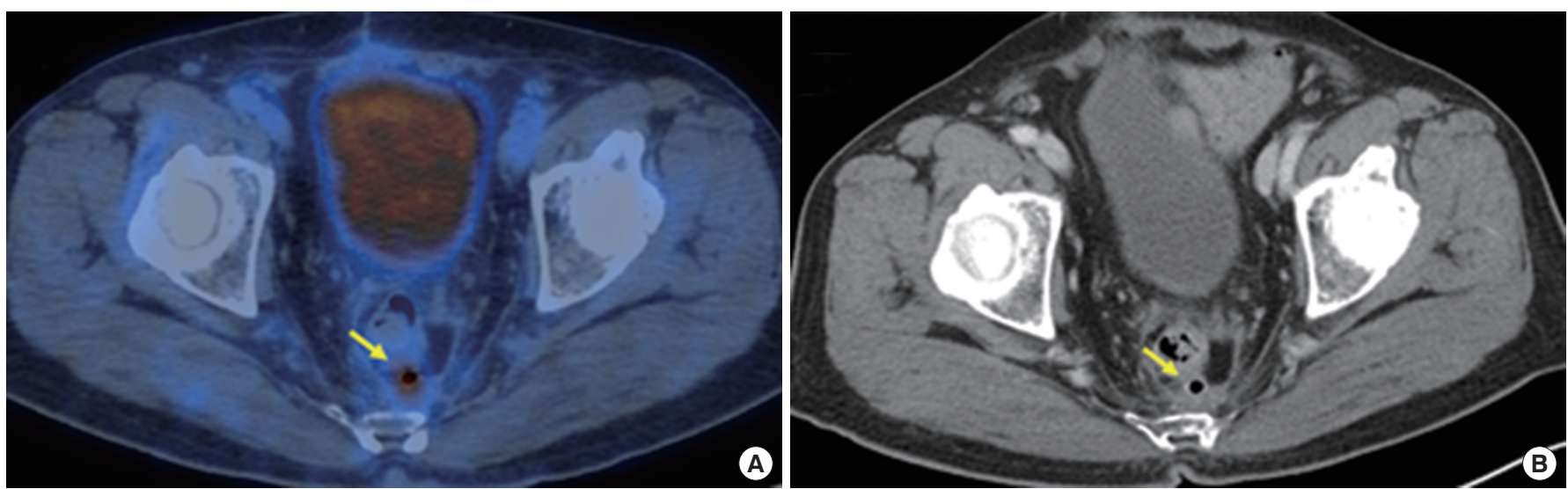

Fig. 4. A 72-year-old male had undergone a laparoscopic anterior resection due to rectal cancer 1 year earlier. (A) Hypermetabolism at the presacral anastomotic sinus (arrow) was shown in the positron emission tomography/computed tomography (CT) fusion image, and (B) an air-containing sinus (arrow) with adjacent fibrosis was shown in the contrast-enhanced CT image. 

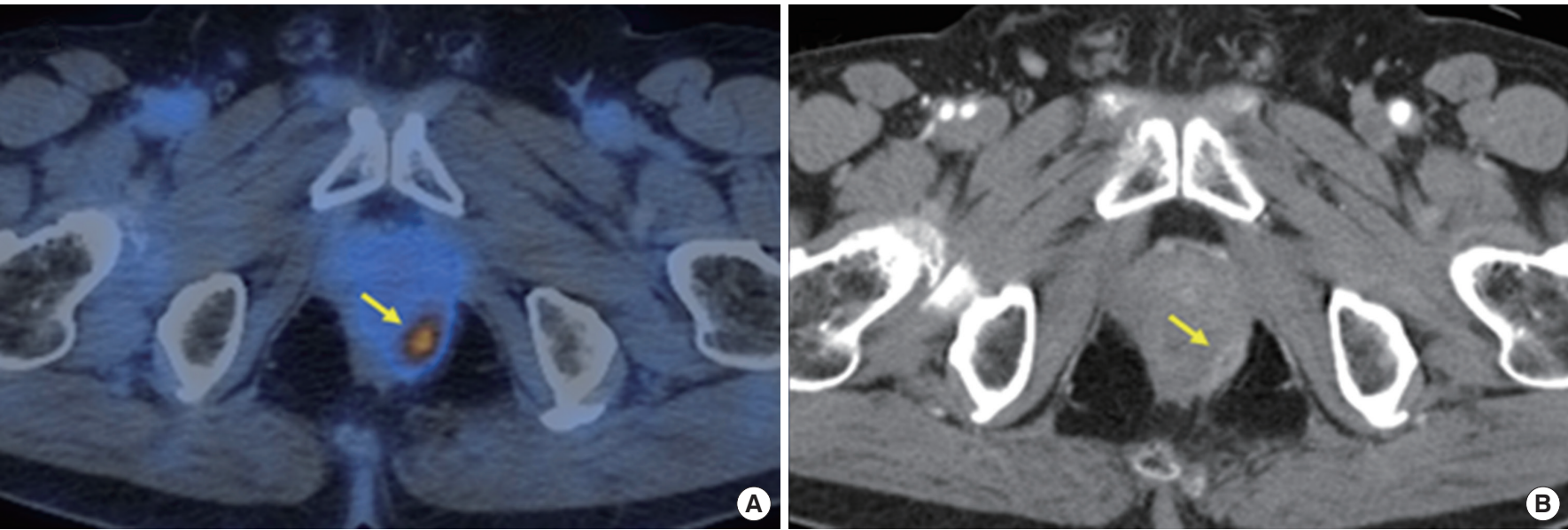

Fig. 5. A 58-year-old male had undergone a laparoscopic low anterior resection due to rectal cancer 4 years earlier. (A) A fistula tract (arrow) in the posterolateral aspect of the anus was observed in the positron emission tomography/computed tomography (CT) fusion image, and (B) an enhancing fistula tract (arrow) was shown in the contrast-enhanced CT image.

the presence of recurrence with no FDG uptake should be considered. Negative PET/CT findings have been reported in patients with histologically mucinous adenocarcinomas $[8,14]$.

In conclusion, PET/CT for postoperative surveillance of patients with rectal and sigmoid colon cancer shows increased FDG uptake not only in areas of local recurrence but also in various categories of benign pelvic lesions. Physicians should be cautious and consider the broad clinical spectrum of possibilities when interpreting such images.

\section{CONFLICT OF INTEREST}

No potential conflict of interest relevant to this article was reported.

\section{REFERENCES}

1. Purandare NC, Dua SG, Arora A, Shah S, Rangarajan V. Colorectal cancer - patterns of locoregional recurrence and distant metastases as demonstrated by FDG PET / CT. Indian J Radiol Imaging 2010;20:284-8.

2. Fiocchi F, Iotti V, Ligabue G, Malavasi N, Luppi G, Bagni B, et al. Role of carcinoembryonic antigen, magnetic resonance imaging, and positron emission tomography-computed tomography in the evaluation of patients with suspected local recurrence of colorectal cancer. Clin Imaging 2011;35:266-73.

3. Zhang Y, Feng B, Zhang GL, Hu M, Fu Z, Zhao F, et al. Value of 18F-FDG PET-CT in surveillance of postoperative colorectal cancer patients with various carcinoembryonic antigen concentrations. World J Gastroenterol 2014;20:6608-14.

4. Sinaei M, Swallow C, Milot L, Moghaddam PA, Smith A, Atri M. Patterns and signal intensity characteristics of pelvic recurrence of rectal cancer at MR imaging. Radiographics 2013;33:E171-87.
5. Tagliacozzo S, Accordino M. Pelvic recurrence after surgical treatment of rectal and sigmoid cancer. A prospective clinical trial on 274 patients. Int J Colorectal Dis 1992;7:135-40.

6. Young PE, Womeldorph CM, Johnson EK, Maykel JA, Brucher B, Stojadinovic A, et al. Early detection of colorectal cancer recurrence in patients undergoing surgery with curative intent: current status and challenges. J Cancer 2014;5:262-71.

7. de Geus-Oei LF, Ruers TJ, Punt CJ, Leer JW, Corstens FH, Oyen WJ. FDG-PET in colorectal cancer. Cancer Imaging 2006;6:S71-81.

8. Makis W, Kurzencwyg D, Hickeson M. 18F-FDG PET/CT superior to serum CEA in detection of colorectal cancer and its recurrence. Clin Imaging 2013;37:1094-7.

9. Khademi S, Westphalen AC, Webb EM, Joe BN, Badiee S, Hawkins RA, et al. Frequency and etiology of solitary hot spots in the pelvis at whole-body positron emission tomography/computed tomography imaging. Clin Imaging 2009;33:44-8.

10. van Koperen PJ, van der Zaag ES, Omloo JM, Slors JF, Bemelman WA. The persisting presacral sinus after anastomotic leakage following anterior resection or restorative proctocolectomy. Colorectal Dis 2011;13:26-9.

11. Weinstein S, Osei-Bonsu S, Aslam R, Yee J. Multidetector CT of the postoperative colon: review of normal appearances and common complications. Radiographics 2013;33:515-32.

12. Lerman H, Metser U, Grisaru D, Fishman A, Lievshitz G, EvenSapir E. Normal and abnormal 18F-FDG endometrial and ovarian uptake in pre- and postmenopausal patients: assessment by PET/CT. J Nucl Med 2004;45:266-71.

13. Nishizawa S, Inubushi M, Okada H. Physiological 18F-FDG uptake in the ovaries and uterus of healthy female volunteers. Eur J Nucl Med Mol Imaging 2005;32:549-56.

14. Schaefer O, Langer M. Detection of recurrent rectal cancer with CT, MRI and PET/CT. Eur Radiol 2007;17:2044-54. 